\title{
The EUV spectrum of the Sun: Irradiances during 1998-2014
}

\author{
G. Del Zanna ${ }^{1}$ and V. Andretta ${ }^{2}$
}

\author{
1 DAMTP, Centre for Mathematical Sciences, University of Cambridge, Wilberforce Road, Cambridge, CB3 0WA, UK \\ e-mail: g.del-zanna@damtp.cam.ac.uk \\ 2 INAF-Osservatorio Astronomico di Capodimonte, Salita Moiariello 16, 80131 Napoli, Italy
}

Received 22 June 2015 / Accepted 14 September 2015

\section{ABSTRACT}

\begin{abstract}
We present calibrated EUV spectral irradiances obtained from observations with the Solar and Heliospheric Observatory (SOHO) Coronal Diagnostics Spectrometer (CDS) Normal Incidence Spectrometer (NIS) during the 1998-2014 period, which spans the cycle 23 maximum and minimum and the cycle 24 maximum. We revise the corrections for the burn-in of the strong lines and our previous long-term calibration of the NIS. We find no indications of further overall degradation of the instrument responsivities after 2010. We compare the CDS irradiances with those obtained by the prototype and flight instruments aboard the Solar Dynamics Observatory (SDO) Extreme ultraviolet Variability Experiment (EVE) and the Thermosphere Ionosphere Mesosphere Energetics Dynamics (TIMED) Solar EUV Experiment (SEE) EUV Grating Spectrograph (EGS). We find overall excellent agreement (to within a relative 10-20\%) with the EVE data (especially during 2010-2012), but point out inconsistencies in some of the prototype and flight EVE irradiances. There is overall agreement with some of the TIMED SEE EGS data. We confirm the small variations in the irradiances of low-temperature lines (except the helium lines) and show that the irradiances in the hot (2-3 MK) lines are significantly lower for the cycle 24 maximum compared to the previous one.
\end{abstract}

Key words. techniques: spectroscopic - Sun: corona

\section{Introduction}

The present paper is part of an on-going effort to provide the best possible solar spectral irradiance in the extreme ultraviolet (EUV). The solar EUV variability causes dramatic changes in the temperature and density of the thermosphere, and it could also have some indirect effects on the climate. Indeed, some of the current global circulation models also require EUV irradiances to properly take the solar forcing into account.

The Solar and Heliospheric Observatory (SOHO) Coronal Diagnostics Spectrometer (CDS; Harrison et al. 1995) Normal Incidence Spectrometer (NIS) has been operating since 1996 until September 2014. The NIS carried out full-Sun scans in its two spectral channels (NIS 1: 308-379; NIS 2: 513-633 Å) during the 1998-2014 period. It is therefore the only instrument capable of providing spectral irradiances in the rising phase of cycle 23.

The long-term degradation of the NIS instrument within the 1996-2010 period was described in Paper I (Del Zanna et al. 2010). The degradation was obtained by assuming that the quiet Sun emission in low-temperature lines as observed with the wide $90^{\prime \prime}$ slit was constant during the cycle. The longterm degradation was combined with the relative responsivities obtained with the line ratio method (Del Zanna et al. 2001) and with the absolute calibration at $584 \AA$ obtained from a NASA/Laboratory for Atmospheric and Space Physics (LASP) EUV Grating Spectrograph (EGS) rocket, flown in 1997 May 15 (Brekke et al. 2000). The assumption of constant radiances was confirmed a posteriori with comparisons with quiet Sun radiances measured in 2007 by the EUNIS sounding rocket (Wang et al. 2011) and the Solar Dynamics Observatory (SDO) Extreme ultraviolet Variability Experiment (EVE, see Woods et al. 2012) prototype (hereafter PEVE), which was flown on 2008 April 14 (Chamberlin et al. 2007, 2009; Woods et al. 2009), although some discrepancies in the strongest lines were noted.
Paper II (Del Zanna \& Andretta 2011) described the methods used to obtain calibrated NIS irradiances from 46 full-Sun radiance measurements during 1998-2010.

Paper III (Andretta \& Del Zanna 2014) analysed in detail the radiances in the main lines. A new limb-brightening function was obtained and shown to be important for the characterisation of the solar radiances. Several new results were obtained.

Paper IV (Del Zanna et al. 2015) compared the CDS irradiances with the count rates measured by the Solar EUV Monitor (SEM) first-order band (SEM 1). Very good agreement was found, except during the cycle 23 maximum, when the SEM 1 count rates are consistently 30-40\% higher than those estimated from the NIS observations.

The aim of the present work has been to extend the CDS calibration and the database of CDS irradiances from the original 46 dates to cover the 1998-2014 period. It was carried out within the SOLID ${ }^{1}$ network, which aims at providing and estimating the solar spectral irradiance across all wavelengths.

Section 2 describes the CDS analysis and calibration. Section 3 briefly presents the irradiances we obtained from SDO EVE and the Thermosphere Ionosphere Mesosphere Energetics Dynamics (TIMED) Solar EUV Experiment (SEE) EUV Grating Spectrograph (EGS) (Woods et al. 2005). Section 4 presents the comparisons, while our conclusions are drawn in Sect. 5 .

\section{The CDS NIS}

The CDS instrument consists of a Wolter-Schwarzschild type-II grazing incidence telescope, a scan mirror, and a set of different slits. The NIS is composed of two normal incidence gratings that disperse the radiation into the NIS detector, mainly

1 http://projects.pmodwrc.ch/solid/ 
composed of a microchannel plate (MCP) and a charge coupled device (CCD). The temporary loss of contact with SOHO in June 1998 degraded the NIS spectra significantly. Its postrecovery line profiles are broadened and asymmetrical and are located in different positions on the CCD.

We first extended the analysis of full-Sun observations from the original 46 dates to a total of 106, covering the 1998-2014 period. We only selected dates when the Sun was relatively inactive, trying to have at least one observation every three months. The CDS as-run database after 2011 was corrupted, and the original FITS files had to be created manually (J. Rainnie, priv. comm.). Unfortunately, in many instances data were either missing or corrupted, so the frequency of the observations varies.

We initially followed the analysis of Paper II. The radiance observations consist of 700 to 1000 single-slit exposures, sampling the whole Sun in about $13 \mathrm{~h}$. Briefly, the analysis involves first de-biasing and removing the cosmic rays. Then, flat-fielding is applied. This includes the correction for the so-called burn-in, which is due to the presence of the MCPs. The exposure to solar radiation with either the $2^{\prime \prime}$ or $4^{\prime \prime}$ slits causes a depression at the core of the lines that need to be corrected for. The NIS spectra are then rotated, and the spectral lines are fitted with Gaussian or post-recovery broadened profiles, removing a background that is mainly due to scattered light. The 69 NIS rasters are then grouped to form a mosaic. The effective area factor is included in this process, since it is dependent on the mirror position. The observations subsample the Sun by a factor of 4 (or 6), so radiances are then simply interpolated. Then, the contribution from the off-limb areas not observed are estimated and added to the totals, to obtain the irradiances. Finally, the responsivities and the long-term calibration factors are applied. For more details, see Paper I.

When we obtained the irradiances and checked the ratios of lines that are known to be relatively constant across a solar cycle, we noted that the irradiances of several bright lines were underestimated by about $30 \%$ during the past few years. We then identified the problem, which was an under-correction of the burn-in. The burn-in has been corrected for in the past by analysing the $90^{\prime \prime}$ observations obtained with the NIMCP study, as described in Thompson (2000). The last corrections were obtained in April 2006. The temporal evolution of the peak of the depressed line profile was modelled with a constant plus exponential decay, and the position and width of the lines with polynomials. In some cases, several components were added. The corrections after 2006 were simple extrapolations of the polynomials.

To obtain a new burn-in correction, we therefore analysed the entire dataset of NIMCP observations, including 46 observations obtained since 2011. We processed the 90" slit exposures by just removing the bias and correcting for the cosmic rays, the flat field, and the slant in the spectra. We considered only the central 141 pixels along the slit. We then averaged the three exposures for each date and inspected the CCD images visually. It has been thought that the exposures centred on active regions would tend to burn the central part (along the slit) in the hot lines such as the Fe XVI 335.4, $360.7 \AA$ doublet, which shows strong burn-in. This would be caused by the instrument being preferentially pointed at the cores of active regions. However, we did not see any obvious evidence for a tendency to burn-in the central part of the slit, so we continued the processing by averaging along the slit. This is really necessary, to remove the strong spatial variations (due to the supergranular network) along the slit.

We then examined the temporal evolution of the spectra and found several problems with the standard correction, in
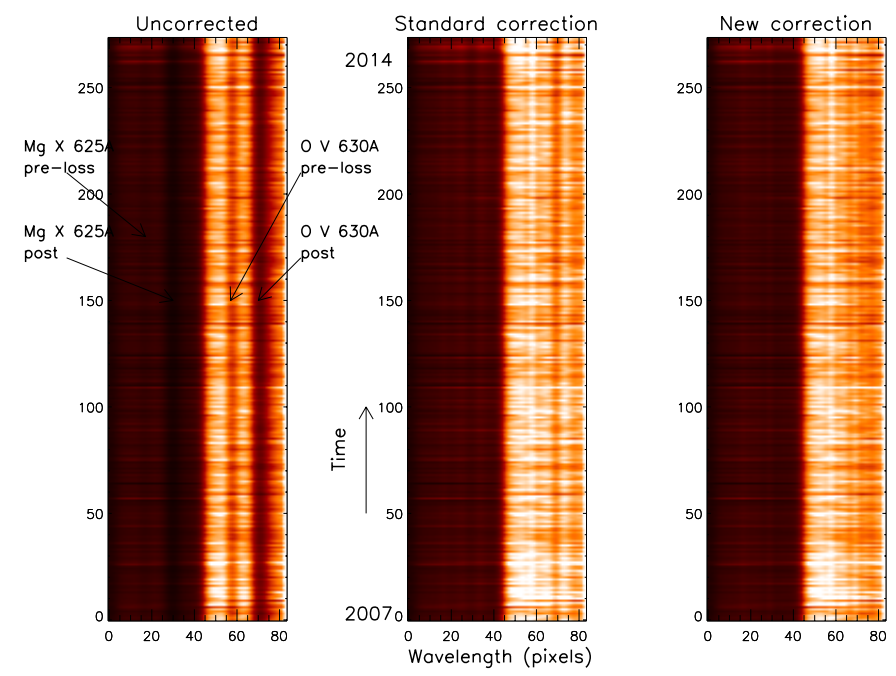

Fig. 1. Portion of the $90^{\prime \prime}$ slit images from 2007 to 2014, showing the pre-loss and post-recovery burn-in in the Mg X 625 and O v $630 \AA$ lines (left), the standard correction (as of April 2006) and the new correction adopted here.

particular for the strong 584.15, 599.7, 625, and $630 \AA$ A lines. The main problem was that the temporal evolution of the peak of the depressed line profile could not be modelled with an exponential, so high degree polynomials had to be adopted instead. The position of several lines within the CCD had some changes and variability but could not be modelled with simple polynomials. The same was often the case for the width.

It turned out that the burn-in correction for the strongest lines becomes much larger than the long-term correction for the past few years. The core of the strong lines is depressed by large factors (3-4), while the long-term correction is at most about a factor of 2. The polynomial extrapolation for dates after 2006 therefore did a pretty good job, considering that it only underestimated the line profiles by about $30 \%$ in some cases.

The absorption profiles in the 90" slit NIMCP study are highly different from line to line and non-symmetrical. The absorption profiles of nearby lines become superimposed and it becomes very difficult to define a correction. The He I 515.0 and 584.15, O III 599.7, Mg X 625, and O V $630 \AA$ A lines are well isolated and we were able to obtain an accurate correction, to say within $10 \%$ or so. An example is given in Fig. 1.

Several weaker NIS 2 lines did not have a significant burnin during the first years so did not have a correction. We have therefore included a new correction for the Ne IV 542.1, 543.9 $\AA$, Ne VI 558.6, 562.8 $\AA$, Ne VII 561.7 $\AA$ lines. For a few other weak lines it was very difficult to find a correction.

In the case of the NIS 1 channel, there were many lines that did not have a correction, and those that did have it required improvement, so we redefined all the post-recovery corrections for all the NIS 1 lines. The process was quite involved.

Once the new burn-in was established, we then looked at the temporal evolution of the corrected $90^{\prime \prime}$ and $4 "$ spectra, and obtained corrections for each wavelength bin from the $90^{\prime \prime}$ data, as done in Paper II. Fig. 2 shows as an example the average radiances in a few NIS 1 wavelength bins, while Fig. 3 shows the same information for selected NIS 2 wavelength bins. In both figures the vertical dashed lines mark a few significant events: the first VDS exposure (January 1996); the first NIMCP data set analysed (May 1996; taken as reference for our subsequent analysis); the date of loss of contact with SOHO (June 1998), 


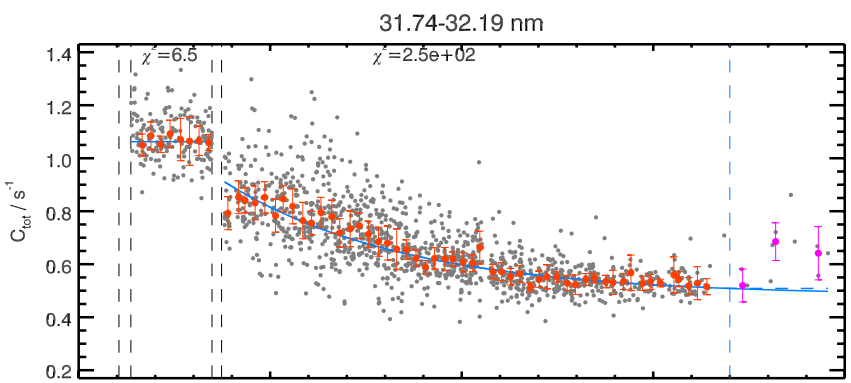

33.83-34.31 nm
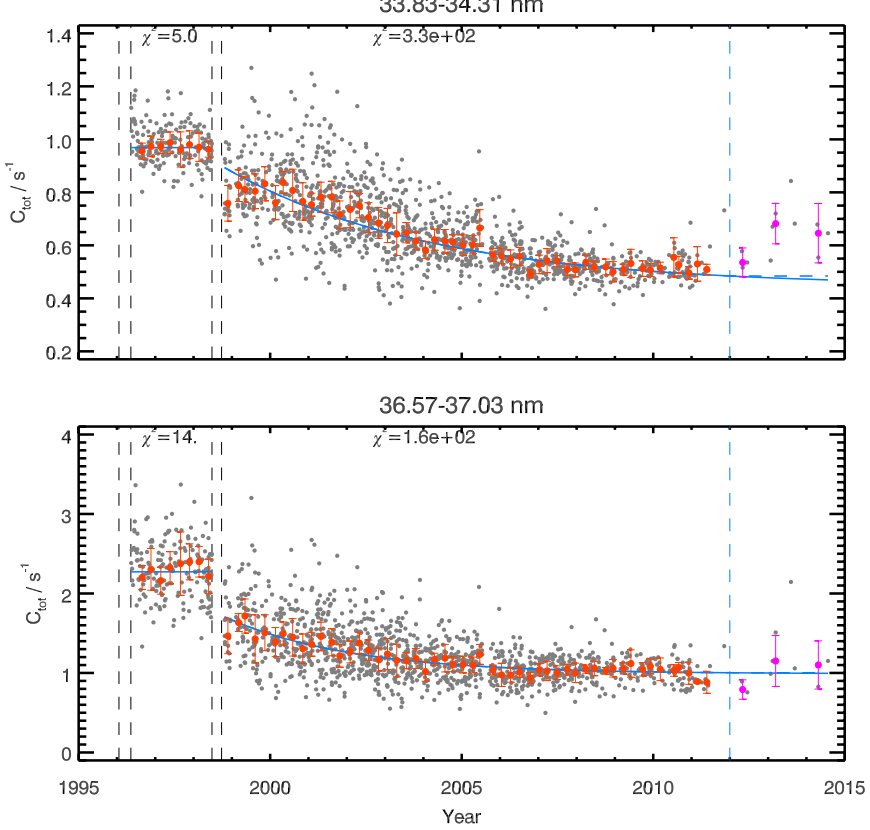

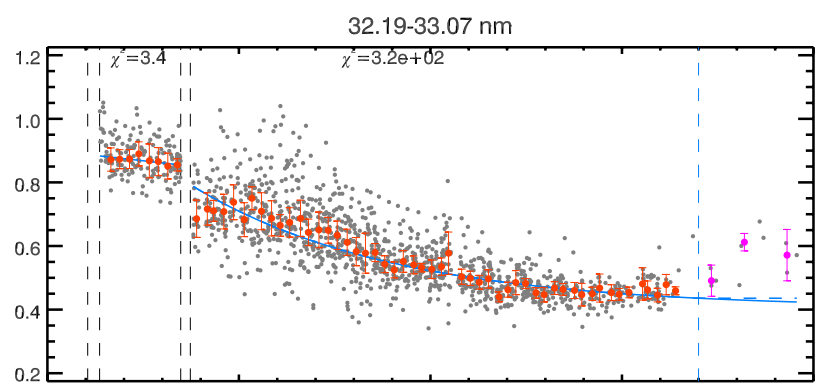

34.31-35.61 $\mathrm{nm}$
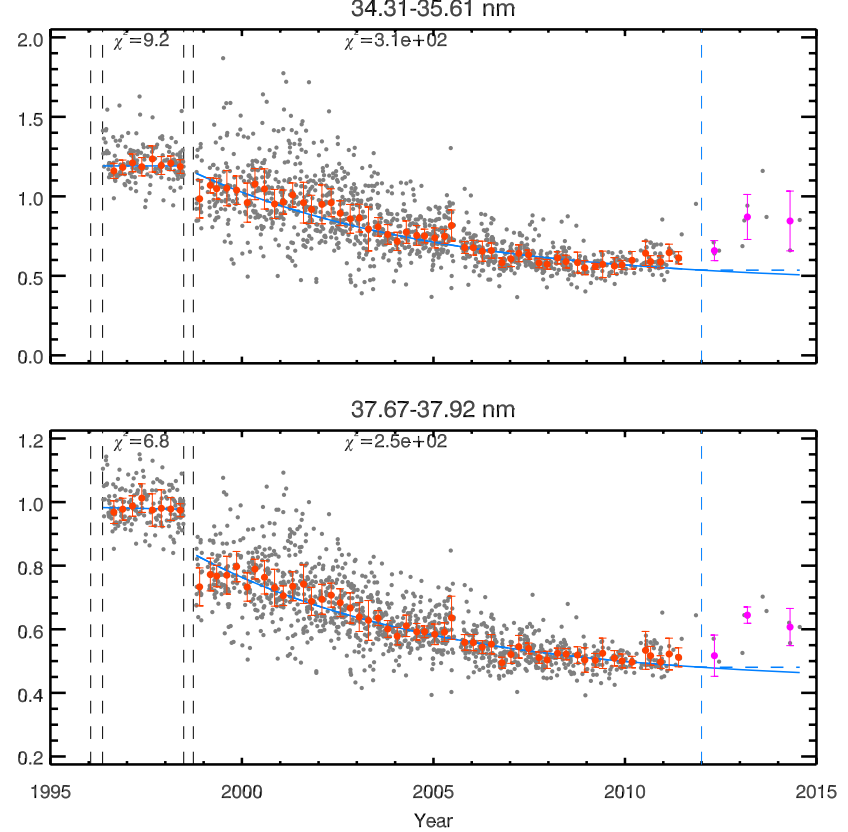

Fig. 2. Time dependence of average radiances (units are photon-events per second) in various wavelength bins from the NIMCP 90" quiet Sun observations in selected NIS 1 wavelength bins. The vertical black dashed lines mark: the first VDS exposure (January 1996), the first NIMCP data set analysed (May 1996; taken as reference for our subsequent analysis), the date of loss of contact with SOHO (June 1998), and the return of SOHO to its normal mode (September 1998). The blue dashed vertical line is the upper limit for the data used for the fit. The radiances of the individual NIMCP data files are shown as grey dots. The 90-day binned data are shown in red. The most recent data (not used for the fit) are shown in magenta. The solid blue line is the fit, while the dashed blue line after 2012 shows the "saturated level".

and the return of SOHO to its normal mode (September 1998). Following the procedure explained in Paper I, the data points were then binned over a period of 90 days, obtaining an estimate of the mean and standard deviation in each time bin (red points and bars in Figs. 2, 3). The wavelength bins are the same as in Paper I, except for the bin between 606.5 and $611.3 \AA$, which we split in two to better take into account the different degradation due to the second order lines Si XI $303 \AA$ and He II $304 \AA$. We experimented alternative corrections, based on quadratic or even higher order polynomial fits to the logarithm of the data, but found that the best fit to the $90^{\prime \prime}$ post-recovery data was given by an exponential + constant fit to the data.

We found no clear signs of further degradation after August 2010. The $90^{\prime \prime}$ spectra actually show an increased background in the past few years, which we think it might be due to an increase in the scattered light, rather than an increase in the sensitivity of the instrument. The quiet Sun count rates of the lines (once the scattered light component has been subtracted) in the 4 " spectra are in fact pretty constant during the past few years. We therefore set the correction value after August 2010 to a constant value, effectively determining a "saturated level" of the correction (horizontal dashed line in Figs. 2, 3).

Once we obtained the new burn-in and long-term corrections, we then reprocessed all the USUN observations, to obtain mosaics of the NIS line radiances for the selected 106 dates. A sample of NIS radiances for a few dates (spanning the cycle 23 maximum, the last extended minimum and the last maximum) is shown in Fig. 4.

We have then totaled the radiances and added an estimate for the not observed off-limb contribution to obtain the irradiances. A (small) correction to convert the observed values at the SOHO location to 1 au was then applied.

As in Paper II, a useful check on the burn-in and long-term correction is given in a few cases by plotting ratios of lines that are known to be relatively constant, independent of the solar activity. Figure 5 shows a few examples, where the constancy of the ratios is satisfactory. The validity of the relative responsivities obtained in Del Zanna et al. (2001) is also clear, since the values of the calibrated ratios are generally in good agreement with previously well-calibrated observations (one exception is the $\mathrm{O}$ III ratio, for which a wide range of values is present).

\section{SDO EVE and TIMED SEE EGS}

We note that SDO EVE observations at moderate resolution are available across a wide wavelength range from May 2010 until May 2014, when the EVE MEGS-A instrument ceased to operate. 

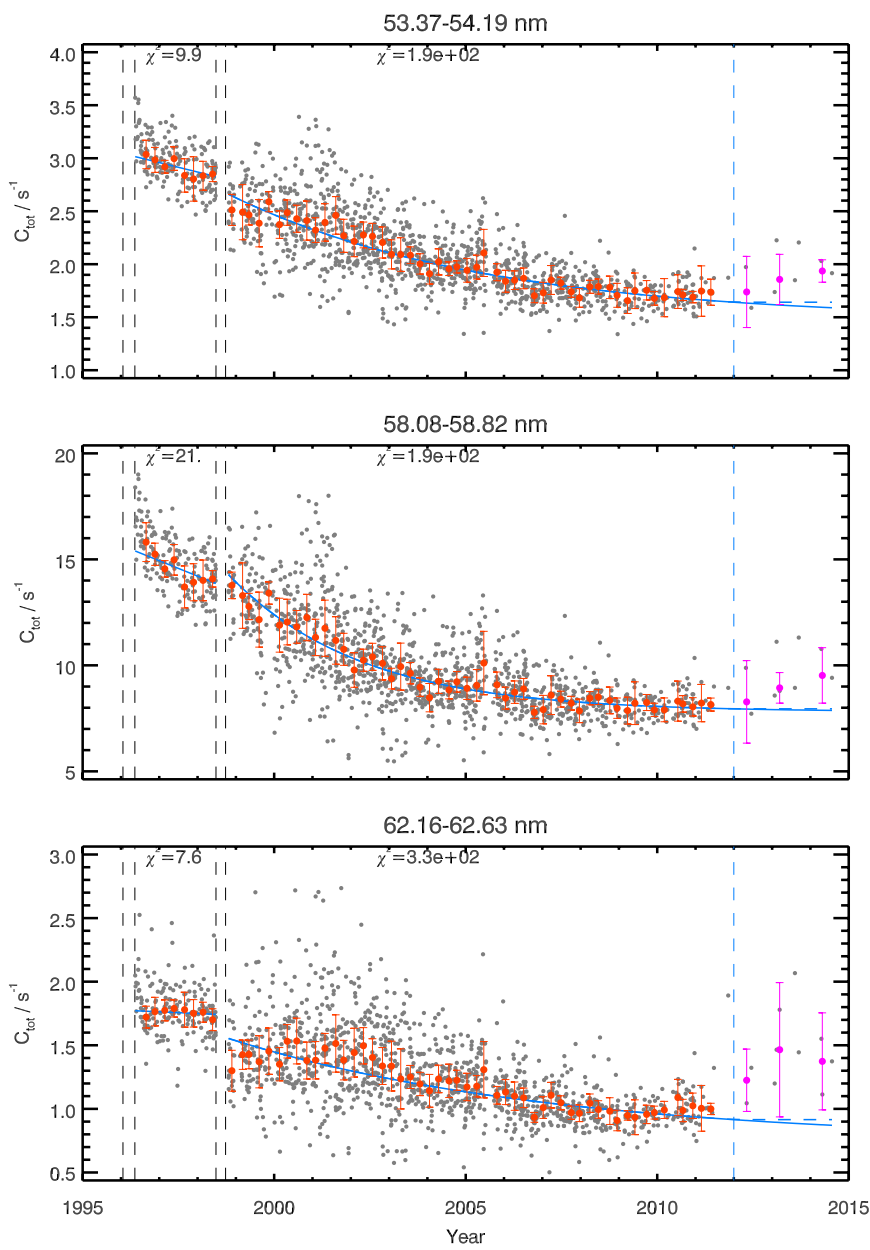
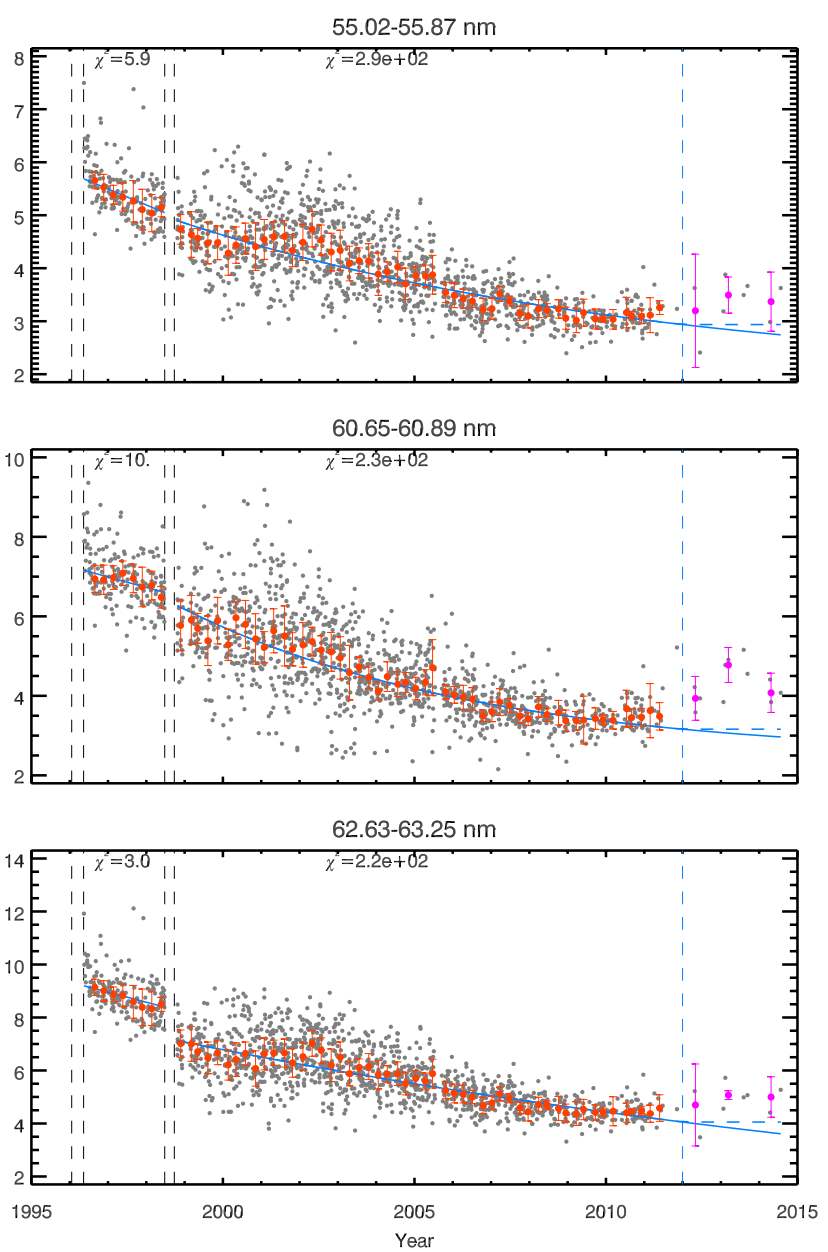

Fig. 3. Same as Fig. 2, for selected NIS 2 wavelength bins.
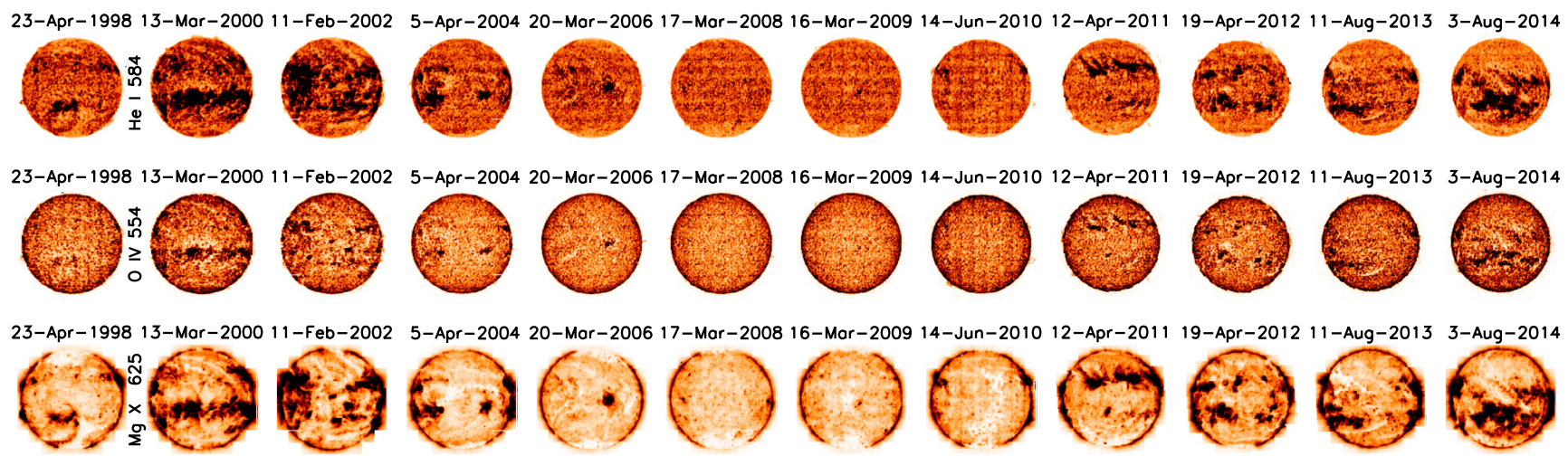

23-Apr-1998 13-Mor-2000 11-Feb-2002 5-Apr-2004 20-Mar-2006 17-Mar-2008 16-Mar-2009 14-Jun-2010 12-Apr-2011 19-Apr-2012 11 -Aug-2013 3-Aug-2014

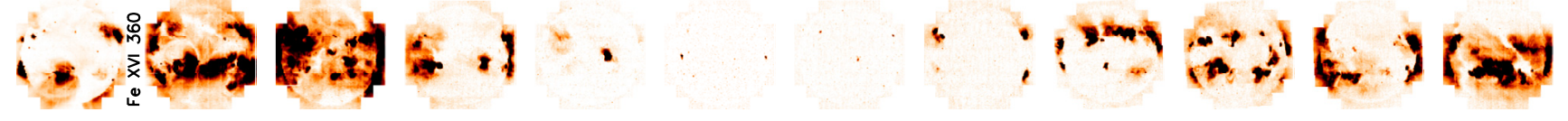

Fig. 4. CDS (negative) calibrated radiances from (top to bottom) He I, O IV ( 0.25 MK), Mg X ( 1 MK), Fe XVI ( 2.5 MK), from 1998 to 2014. Images are on linear intensity scales, the same for each line.

The degradation of the EUV instruments is a notoriously serious problem (see the recent review by BenMoussa et al. 2013). The SDO EVE, as all EUV instruments, suffered a significant degradation, and its calibration is continuously being revised with a combination of methods. These methods include observations with different front filters and comparisons with observations of a clone on sounding rocket flights. Version 4 (released in 2014) also introduced our line ratio method (Paper I) to adjust the relative degradation of many spectral lines. We note that differences between the various versions of the EVE calibration are significant (of the order of 50\%) at some wavelengths. A further improvement in the EVE calibration was introduced at the end of 2014 with v.5: this mostly affected the irradiances in some of the lines in 2014-2015. 

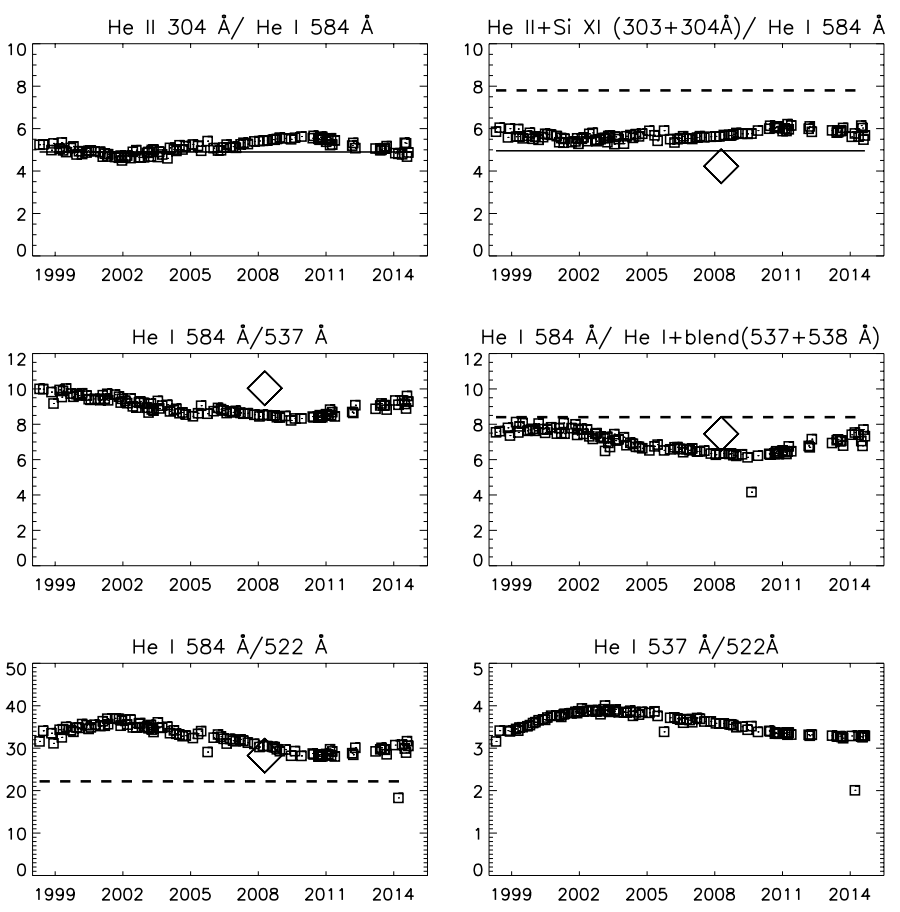

O III $599.6 \AA / 525.8 \AA$
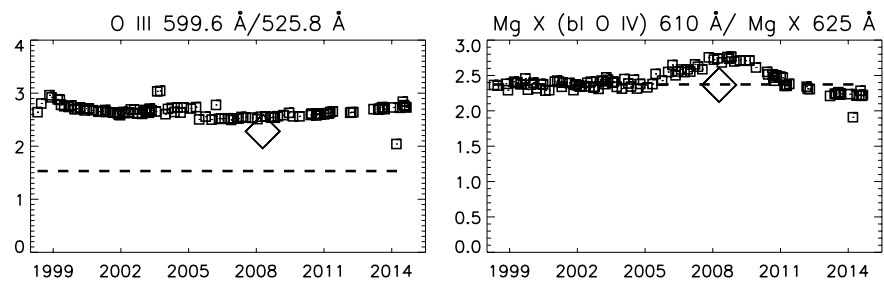

Fig. 5. Ratios (photon units) of SOHO/NIS EUV irradiances in lines from $\mathrm{He}$ and other ions which should be approximately constant with the solar cycle. The dashed lines correspond to the values from the averaged Skylab ATM radiances in the quiet Sun, while the diamond refer to the SDO/EVE prototype spectrum of 2008 April 14. The full line indicates the irradiance ratios from the Heroux et al. (1974) measurements.

We analysed the version 5 daily-averaged EVE level 3 merged spectra (available at LASP), by fitting the lines with Gaussian profiles, removing a background. The background subtraction affects the irradiances of some of the lines slightly, by approximately $10 \%$. The EUV background is a superposition of hydrogen and helium continua and of the forest of weak lines that are visible in high-resolution spectra. To show how this background subtraction affects the lines, we plot the irradiances provided by the EVE team in Fig. 6 (the values tagged as LINE_IRRADIANCE in the data products) as obtained by integrating the lines without subtracting a background.

We note that v.5 improves agreement with our CDS results for several lines, although some discrepancies remain, as discussed below. We also note that the profiles of some of the strong EVE lines are not Gaussian, for example O v $630 \AA$ A. For some of the EVE lines, the profiles were Gaussian at the beginning of the mission, but then have with time become non-Gaussian. For the following isolated lines, we have therefore obtained their irradiance by summing the pixel intensities: O IV $554 \AA$, He I $584 \AA$, Mg X $610 \AA$, and O v $630 \AA$. Among these lines, integrating the line profiles instead of Gaussian fitting increases significantly the irradiance only for the OV $630 \AA$.

The uncertainties due to the statistics in the count rates are very small. The main uncertainty lies in the radiometric calibration of the EVE calibration rocket flights at NIST and in the way that is transferred to the EVE flight data. To provide an estimate of the uncertainty in the EVE irradiances, we show the NIST combined standard uncertainty in Fig. 6 (the values tagged as LINE_ACCURACY in the data products) as provided by the EVE team. This quantity is of the order of $10 \%$ with some time variability.

We also obtained the irradiances of the main lines in the TIMED SEE EGS spectra. We used the EGS level 2 merged dataset version 11 as available at LASP (“see_egs_L2_merged_2014295_011.ncdf”).

\section{Results and comparisons}

Figure 6 shows our final NIS irradiances for a selection of lines. The overall uncertainty in the CDS data is estimated to be approximately $20 \%$. We only show the uncertainty bars for the first, the last and the middle measurements to simplify the plots. The CDS irradiances are compared with the results of the sounding rocket EGS of May 1997 by Brekke et al. (2000) (asterisks), the TIMED SEE EGS (triangles), and the SDO/EVE data. The small diamonds refer to our background-subtracted measurements, while the $\times$ (labelled EVE std) show the line irradiances without background subtraction as provided by the EVE team, with their uncertainties, whenever available.

The large diamond shows the irradiance measured from the EVE prototype, which was flown on 2008 April 14. The uncertainty on the measurement is taken from the uncertainty in the ground calibration, as estimated by Chamberlin et al. (2009).

The NIS observes the Si XI $303 \AA$ and He II $304 \AA$ lines in second order. The He II line is the most prominent in the EUV; however, as we pointed out in Paper II (Del Zanna \& Andretta 2011), its calibration was incorrect by large factors in most previous observations, and a new calibration was suggested. For a direct comparison with the lower resolution SDO EVE and TIMED SEE EGS data, we summed the Si XI and He II irradiances, shown in Fig. 6. Very good agreement (within 10-20\%) is found between our irradiances and the PEVE and EVE ones. On the other hand, the EGS irradiances are (still) significantly lower and are therefore not reliable.

We recall (see Paper IV, Del Zanna et al. 2015) that our NIS irradiances are also in excellent agreement (within 10\%) with the broad-band SEM 1 observations, except for the cycle 23 maximum, where the SEM 1 count rates are consistently higher (by $30-40 \%$ ) than those estimated from the NIS observations.

The second strongest EUV line is the He I $584.15 \AA$ one. Its ratio with the He II $304 \AA$ line is relatively constant (see Fig. 5) as expected, so its NIS irradiance should be correct. Very good agreement is found with the EGS irradiances until the end of 2011, when the EGS values decrease, which is unreasonable. Relatively good agreement is also found with the EVE 2010 and 2011 data; however, the EVE values increase to unreasonable levels during the cycle 24 maximum in 2014 (higher than those of the cycle 23 maximum). It is also interesting to note that the large value of the irradiance measured by PEVE in 2008 during the minimum is clearly incorrect, being inconsistent with our NIS and the EVE measurements in 2010 (when the Sun was still close to minimum).

The increase in the EVE irradiances of the He I 584.15 A line is probably caused by an over-correction of the degradation of this strong line in the EVE data. In fact, good agreement is found between our NIS irradiances and the EVE ones for the next two 
He II (bI Si XI) $304 \AA$
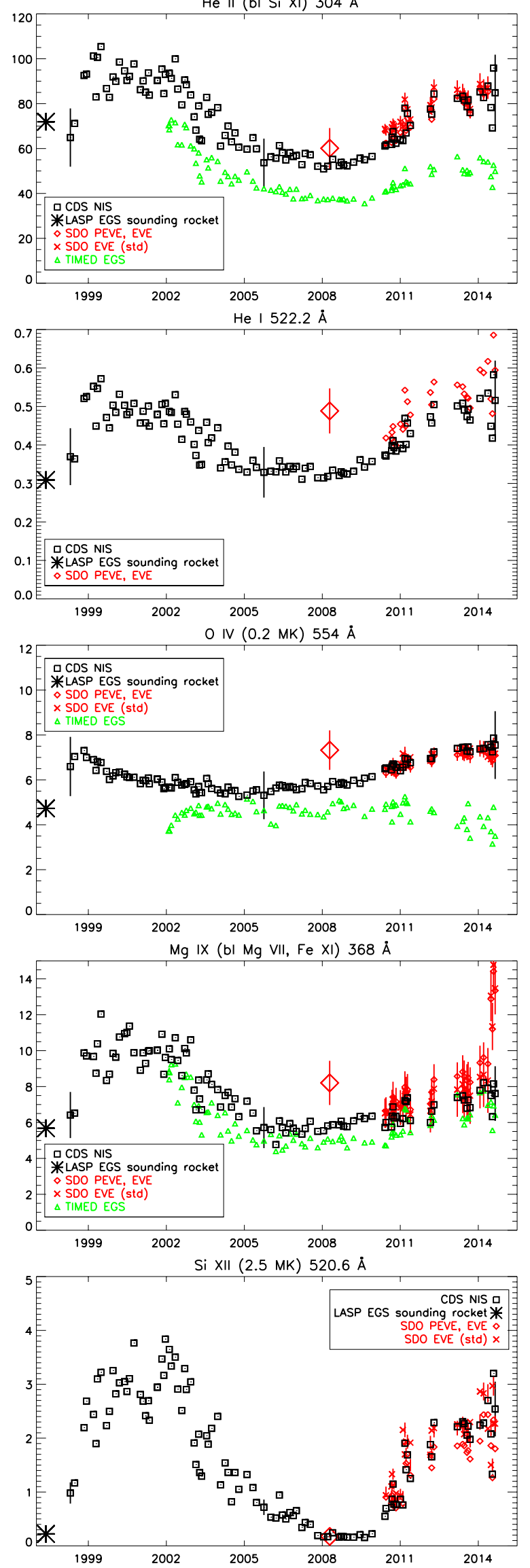

He I $584 \AA$
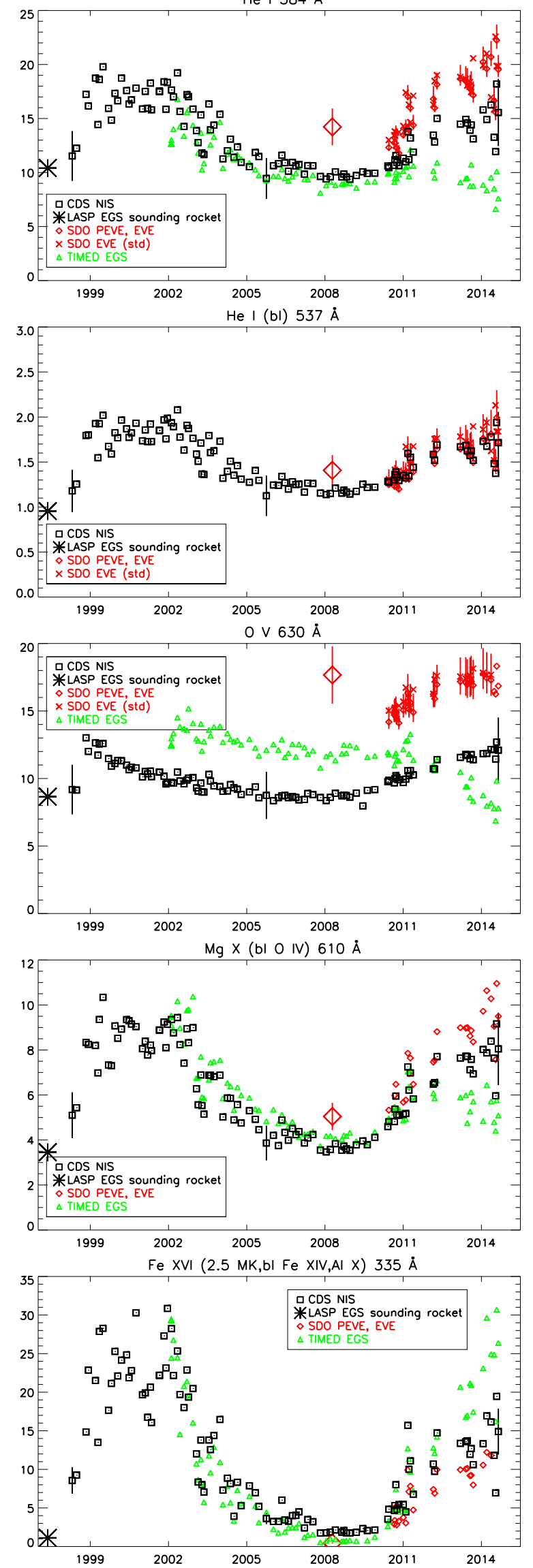

Fig. 6. SOHO CDS NIS irradiances (boxes) at 1 au $\left(10^{8}\right.$ phot $\left.\mathrm{cm}^{-2} \mathrm{~s}^{-1}\right)$ for cycle 23 and 24 , compared to other irradiances. 
lines in the series, the 537 (which is blended but stronger) and the $522 \AA$ ones, as shown in Fig. 6 (second row).

The third strongest EUV line is the OV $630 \AA$, formed in the transition region. As shown for the first time in Paper II, the irradiances of TR lines vary very little with the solar cycle, and indeed the behaviour of the $\mathrm{OV}$ line follows the general trend. The EGS data also show a similar trend until 2011, after which the EGS calibration is clearly in need of revision. The EVE irradiances show a clear increase which seems unreasonable, and probably caused, as for the He I $584.15 \AA$ line, by an over-correction of the EVE degradation. There is a clear offset between the NIS and the EGS/EVE irradiances; however, it is not as large as the offset in the 2008 irradiance measured by PEVE. This line is at the edge of the NIS 2 channel and it is unclear if the NIS responsivity is correct.

The next strongest TR EUV line is the O IV multiplet at $554 \AA$ A. We find excellent (within 10\%) agreement with the EVE irradiances, but not with the PEVE one. As in other cases, the PEVE irradiance is higher, at odds with all the other irradiances. Relatively good (within 20\%) agreement is found with the EGS data, but again only until 2011 (cf. Fig. 6).

Within the NIS 1, the strongest line is the Mg IX, formed at coronal temperatures, around $1 \mathrm{MK}$. This line, at the EVE or EGS resolution, is severely blended. Taking the blends into account, we find relatively good agreement between the EGS and EVE irradiances, but again the PEVE irradiance appears overestimated (cf. Fig. 6).

Another strong EUV line is the Mg X $610 \AA$, blended with O IV. Very good agreement is found with the EVE and EGS irradiances (until 2011), while again the PEVE measurement is higher (cf. Fig. 6). The same occurs for the weaker component of the $\mathrm{Mg}$ X doublet at $625 \AA$ (not shown). There is a tendency in the EVE data to overestimate the irradiance after 2011.

Finally, the hottest (2-3 MK) lines for which irradiances are measurable by NIS are the Si XII $520.7 \AA$ and the Fe XVI 335.3, $360.8 \AA$ doublet. The Si XII line is blended in EGS spectra so we cannot show the EGS measurements; however, we find good agreement with the EVE and PEVE measurements (cf. Fig. 6, bottom row). We also note that our irradiances clearly show that the cycle 24 maximum is much smaller than the previous one. This is in agreement with the overall sunspot numbers and the radio flux F10.7. Indeed, there is a very good correlation between the radio flux and the irradiance of this line, as shown in Paper II. Similar results hold for the Fe XVI doublet. For the weaker line, we find very good agreement with the PEVE and EVE irradiances (the line is not visible at the EGS resolution). The stronger Fe XVI $335.3 \AA$ line is severely blended at the EVE and EGS resolution. Taking the blends into account, we find good agreement with the EGS irradiances until 2012, after which the irradiance increases to unreasonable levels (cf. Fig. 6, bottom row). Good agreement is found with the PEVE and EVE irradiances, although some unreasonably low EVE values around 2013 are present.

\section{Conclusions}

We have provided here our final effort on the CDS NIS irradiances $^{2}$. It has been a long journey. We planned and obtained the first measurements for the calibration of the relative

\footnotetext{
2 Our new burn-in and revised long-term correction will be made available via SolarSoft, while the calibrated irradiances will be available via the SOLID web pages.
}

responsivities during 1996-1998. Full details were presented in Del Zanna (1999), and a summary published in Del Zanna et al. (2001). We recall that our responsivities were relative to the responsivity at the He I $584 \AA$ line $\left(4.75 \pm 0.4 \times 10^{-4}\right.$ events/photon $)$ obtained by Brekke et al. (2000) from a LASP EGS sounding rocket flight, which was radiometrically calibrated on the ground. The validity of our responsivities has been confirmed not only in terms of good agreement with previously measured ratios, but now also with the well-calibrated EVE spectra.

The agreement between our NIS irradiances and the EVE ones cannot be a coincidence, because it holds for strong and weak lines, and the two instrument have been calibrated in a completely independent way. The agreement is incredibly good, if one considers the intrinsic difference between the observations, and all the corrections applied to the data. Some of these corrections were large. By 2010, when EVE started the observations, the NIS had degraded its responsivity by a factor of two, and the strong lines were burned-in by even larger factors.

We note that our recommended CDS responsivities are different than those provided by the ground calibration or its subsequent revisions (see the history of the CDS calibration in the Appendix of Paper I), although they differ from those of the latest revision at most by $30 \%$. We also note that our revised longterm correction is quite similar to the previous one, which is being used for the final calibration of the entire CDS NIS database.

When we compared in Paper I (Del Zanna et al. 2010) our NIS irradiances, obtained with a new estimate of the long-term correction, to those measured by the SDO EVE prototype, we found overall good agreement, although the irradiances of several of the strongest lines were much higher in the EVE prototype spectra. We were puzzled because the EVE spectra were calibrated before and after the flight, and should therefore have been reliable. However, comparison with the flight EVE data, our revised NIS irradiances and the TIMED SEE EGS ones suggests that some of the prototype EVE irradiances were overestimated. The solar minimum reference EUV spectrum is therefore not quite the same as observed by the prototype EVE.

It is also clear that the corrections for the EVE degradation after 2012 need further improvement. Given the poor (and degrading) spectral resolution of the TIMED SEE EGS, we consider the relatively good agreement with our NIS measurements satisfactory, although the EGS calibration is clearly in need of a revision for dates after 2011.

The various comparisons clearly indicate that our NIS irradiances are the most reliable EUV irradiances for cycle 23 and 24. The uncertainties in the various calibration factors, together with these comparisons, suggest for the NIS calibration an overall uncertainty of $20 \%$ across the entire period of observations. We consider this to be a significant achievement.

For its endurance, the NIS instrument has turned out to be the best solar EUV spectrometer flown in space. The EVE grazing incidence spectrograph (MEGS-A) unfortunately ceased to operate in May 2014, so future instruments are needed to continue the monitoring of the EUV solar irradiance.

Acknowledgements. A significant part of the present work was carried out by G.D.Z. within SOLID. SOLID (First European SOLar Irradiance Data Exploitation) is a collaborative SPACE Project under the Seventh Framework Programme (FP7/2007-2013) of the European Commission under Grant Agreement No. 313188. G.D.Z. also acknowledges support by the UK STFC via the consolidated grant of the DAMTP astrophysics group at the University of Cambridge. V.A. acknowledges partial support by the Italian Space Agency (ASI) through ASI-INAF agreement I/013/12/0. We acknowledge support to participate to two workshops on the intercalibration of EUV instruments, held at the Royal Observatory of Brussels, partially funded by STCE. We would like to thank J. Rainnie for providing the CDS FITS files for 2011 onwards. 
CDS was built and operated by a consortium led by the Rutherford Appleton Laboratory (RAL), which included UCL/Mullard Space Science Laboratory, NASA/ Goddard Space Flight Center, Max Planck Institute for Extraterrestrial Physics, Garching, and Oslo University. The results obtained here could not have been achieved without the efforts of a large number of operation and scientific staff which have successfully run the CDS instrument at STFC/RAL (UK) and NASA/GSFC (US). SOHO is a mission of international cooperation between ESA and NASA.

\section{References}

Andretta, V., \& Del Zanna, G. 2014, A\&A, 563, A26

BenMoussa, A., Gissot, S., Schühle, U., et al. 2013, Sol. Phys., 288, 389

Brekke, P., Thompson, W. T., Woods, T. N., \& Eparvier, F. G. 2000, ApJ, 536, 959

Chamberlin, P. C., Hock, R. A., Crotser, D. A., et al. 2007, in SPIE Conf. Ser., $6689,66890 \mathrm{~N}$
Chamberlin, P. C., Woods, T. N., Crotser, D. A., et al. 2009, Geophys. Res. Lett., 36,5102

Del Zanna, G. 1999, Ph.D. Thesis, Univ. of Central Lancashire, UK

Del Zanna, G., \& Andretta, V. 2011, A\&A, 528, A139

Del Zanna, G., Bromage, B. J. I., Landi, E., \& Landini, M. 2001, A\&A, 379, 708

Del Zanna, G., Andretta, V., Chamberlin, P. C., Woods, T. N., \& Thompson, W. T. 2010, A\&A, 518, A49

Del Zanna, G., Andretta, V., Wieman, S., \& Didkovsky, L. 2015, A\&A, 581, A25

Harrison, R. A., Sawyer, E. C., Carter, M. K., et al. 1995, Sol. Phys., 162, 233

Heroux, L., Cohen, M., \& Higgins, J. E. 1974, J. Geophys. Res., 79, 5237

Thompson, W. T. 2000, Opt. Eng., 39, 2651

Wang, T., Thomas, R. J., Brosius, J. W., et al. 2011, ApJS, 197, 32

Woods, T. N., Eparvier, F. G., Bailey, S. M., et al. 2005, J. Geophys. Res. (Space Phys.), 110, 1312

Woods, T. N., Chamberlin, P. C., Harder, J. W., et al. 2009, Geophys. Res. Lett., 36,1101

Woods, T. N., Eparvier, F. G., Hock, R., et al. 2012, Sol. Phys., 275, 115 